\title{
Meat Food Intolerance-Prevalence among the Bulgarian Population, Main Characteristics and Health Significance
}

\author{
Adriana Agovska* and Maria Nikolova* \\ Assistant Professor, Department of Epidemiology and Hygiene, Medical University-Sofia, Bulgaria
}

*Corresponding authors: Adriana Agovska, Assistant Professor, Department of Epidemiology and Hygiene, 2 Zdrave, Medical University-Sofia, Bulgaria, Tel: +359-888 775284; E-mail: aiabg@yahoo.com

Maria Nikolova, Assistant Professor, Department of Epidemiology and Hygiene, 2 Zdrave, Medical University-Sofia, Bulgaria, Tel: +359-889 628851; E-mail: marianikolova@mail.bg

Received: 11 Oct, 2021 | Accepted: 01 Dec, 2021 | Published: 07 Dec, 2021

Citation: Agovska A, Nikolova M (2021) Meat Food Intolerance-Prevalence among the Bulgarian Population, Main Characteristics and Health Significance. J Epidemiol Public Health Rev 6(4): dx.doi.org/10.16966/2471-8211.219

Copyright: @ 2021 Agovska A, et al. This is an open-access article distributed under the terms of the Creative Commons Attribution License, which permits unrestricted use, distribution, and reproduction in any medium, provided the original author and source are credited.

\section{Abstract}

Introduction: Meat food intolerance is a current problem but studies regarding its distribution and features in Bulgarian have not yet been conducted. The aim of this study is to establish the prevalence of meat intolerance, main characteristics, and some correlations with other food's intolerances.

Objectives: A total of 1579 healthy people over 18 years old, were surveyed. From all studied, the cases of meat intolerance were separated and they were included in this study for correlations with other foods intolerances.

Method: Food Detective-CNS (Cambridge Nutrition Sciences Ltd) to identify the foodstuffs to which the body exhibits intolerance. The bases of the study are the forming antibodies of class IgG-markers for intolerance to different food groups and food products. Individuals were tested for 46 foods, incl. 4 types of meat-pork, chicken, lamb, veal.

Results: $6.2 \%$ of those tested showed intolerance to meat, most often to pork-37.7\%, followed by chicken-25.6\%, to lamb-22.4\% and the least to veal-14.3\%. Correlation relationships between the presence of meat intolerance (total for all types of meat and by individual species) were determined and analyzed, with the highest correlation with egg intolerance $(p<0.0001)$, to cereals $(p<0.0001)$ and to milk ( $p<0.0001)$, to a lesser extent to vegetables $(p=0.0079)$ and with no statistical significance for nuts and gluten $(p>0.001)$.

Conclusion: There are many other correlations analyzed for different types of foods. The results of the study are important in the nutrition practice: prevention and treatment of nutritional diseases, overweight and other eating disorders, creation of a suitable individual diet, restrictions on the use of particular food groups or products, etc.

Keywords: Food (meat) intolerance; Food specific IgG antibody; Correlations between food products

\section{Introduction}

Food intolerance is a widespread condition in the global populationit is estimated that between 5 to $45 \%$ of the people could be affected $[1,2]$. The problem is also topical for Bulgaria, but so far no studies have been conducted on the prevalence of IgG based intolerance to various foods. There is also no data on meat intolerance and our study is aimed at establishing the prevalence and main characteristics of nutritional intolerance towards meat in Bulgaria. The presented data are part of a comprehensive epidemiological study on the prevalence of food intolerances among the Bulgarian population.

As an immunological identification, food intolerance is associated with an IgG class of antibodies in opposite to food allergy-which is most often mediated by IgE antibodies [3]. Enzymatic insufficiency and reactions resembling chronic nutritional intoxication are often present. Important is the fact that in food intolerant people it is possible to detect allergic symptoms and IgG antibodies to certain foods instead of the food-specific IgE antibodies [4]. This sometimes makes nutritional intolerances and food allergies difficult for differential diagnosis.

IgG-mediated food intolerance has been associated with a wide range of specific and nonspecific symptoms-allergy-related symptoms (increased irritability, rashes, urticaria and asthma $[5,6]$, gastrointestinal symptoms suggestive of irritable bowel syndrome, chronic fatigue and hair loss $[2,7,8]$, neurological manifestations such as migraine $[9,10]$. There are also manifestations of post-feeding drowsiness, bloating, feeling of heaviness in the stomach, diarrhea, constipation, stomach acids, vomiting, muscle and joint pain, sneezing, rashes, lethargy and a generally feeling unwell, as well as a combination of several symptoms. Anti-food IgG antibodies are tightly associated with systemic low grade inflammation and may 
contribute to the development of obesity, insulin resistance, diabetes mellitus and atherosclerotic vascular disease [11].

Reactions occur after many more hours, even days, than food allergies. A ban on consuming "unbearable" food leads to a significant improvement in the evaluation of symptoms $[6,8,10]$. The most widespread and well-studied are nutritional intolerance to gluten (gluten enteropathy-celiac disease), to fructose, lactose and histamine intolerance. There is often intolerance to several foods at the same time.

Some less common food intolerances, such as meat, are of interest. Meat and meat products are widely distributed foods in Bulgaria, but there are no studies on the prevalence of meat intolerance and correlation with other intolerances in the Bulgarian population.

\section{Materials and Methods}

\section{Study design}

Our study is establishing the prevalence (frequency, distribution) of meat intolerance, some characteristics and correlations with other food's intolerances. The data are part of a study on the incidence and prevalence of food intolerance for 46 major foods (Table 1). We can determine that this is a "pilot" study that shows the main characteristics and trends of food intolerance, which has not been studied in Bulgaria.

\section{Study participants}

A total of 1579 people over 18 years of age were examined. The subjects came voluntarily, at their own request, for consultation with a nutritionist for advice and/or prescription of a proper diet. They all visited the dietitian's office at their own will.

The subjects are clinically healthy, with diverse BMI-with normal, overweight and obesity. All subjects were explained the goals and objectives associated with the study. All surveyed participants were provided in advance with detailed information about the type of study, its nature and the way of realization of the established results.

The subjects had the opportunity to ask questions and receive a comprehensive answer to all questions and to receive an individual consultation with a specialist in "nutrition and dietetics".

From all studied, the cases of meat intolerance were separated and they were included in this study for correlations with other foods/ products. The persons with meat intolerance are 98, respectively 15 men and 83 women.

\section{Study instrument (method)}

A blood test is used-Food Detective IgG Food Intolerance Test-CNS (Cambridge Nutrition Sciences Ltd) [12] to identify the foodstuffs to which the body exhibits intolerance. The bases of the study are the forming antibodies of class IgG, markers for intolerance to different food groups (and food products). The test is performed by a blood sample from the test person's finger. Different types of nutritional extracts are used to identify foods to which antibodies are produced (foods that induce the production of antibodies in a particular blood sample). The test is reliable and informative for the presence of intolerance to the examined foods.

Individuals were tested for 46 foods shown in table 1. This Food intolerance tests are safe, easy to perform. All subjects were explained the type of test, characteristics of test, test safety.

Food-specific IgG antibodies were detected using a Microarray test for food specific IgG antibodies (Genarrayt, Omega Diagnostics Group, Scotland, United Kingdom) that measures IgG levels against 46 food substances using a single blood sample.

\section{Statistics (statistical analyses)}

All collected data were analyzed using IBM SPSS version 21 (New York, USA statistical package). Descriptive characteristic of the studied sample was evaluated by determining the percentages and mean \pm Standard Deviation (SD). The Chi-square test was used for comparison of proportions. Comparison of the relative shares of food intolerance of 4 types of meat: pork, chicken, lamb and veal, and the other tested products. Variation, correlation analysis, test $\chi^{2}$ (chisquared) and cross tables were used. A p-value $\mathrm{p}<0.05(<0.001)$ was considered statistically significant.

\section{Results}

The study results show IgG-mediated meat intolerance in $6.2 \%$ of the subjects studied. This equals to 98 people, including 15 men and 83 women. The majority of people with meat intolerance- $92.8 \%$ (90 people) show intolerance to one kind of meat. Combined intolerance to more than one kind of meat is found in 8 people- $7.2 \%$. Prevalence of meat intolerance among subjects is shown in figure 1 .

Table 1: List of foods used in the study.

\begin{tabular}{|c|c|c|c|}
\hline 1. Belt & 2. Wheat & 3. Rice & 4. Corn \\
\hline 5. Rye & 6. Wheat / paste, c./ & 7. Gluten & 8. Almonds \\
\hline 9. Brazil nuts & 10. Cashew & 11. Tea & 12. Walnuts \\
\hline 13. Milk & 14. Eggs & 15. Chicken meat & 16. Lamb meat \\
\hline 17. Veal meat & 18. Pork meat & 19. White fish-cod, flounder & $\begin{array}{l}\text { 20. Freshwater fish-salmon, } \\
\text { trout }\end{array}$ \\
\hline 21. Tuna & $\begin{array}{l}\text { 22. Fruits-shrimps, crabs, } \\
\text { lobsters, mussels }\end{array}$ & 23. Broccoli & 24. White cabbage \\
\hline 25. Carrots & 26. Leek & 27. Potatoes & 28. Celery \\
\hline 29. Cucumber & 30. Peppers-red, green, yellow & $\begin{array}{l}\text { 31. Beans-peas, lentils, } \\
\text { beans }\end{array}$ & 32. Grapefruit \\
\hline 33. Melon and watermelon & 34. Peanuts & 35. Soy & 36. Cocoa \\
\hline 37. Apple & 38. Currant & 39. Olives & 40. Oranges and lemons \\
\hline 41. Strawberries & 42. Tomatoes & 43. Ginger & 44. Garlic \\
\hline 45. Mushroom & 46. Yeast & & \\
\hline
\end{tabular}




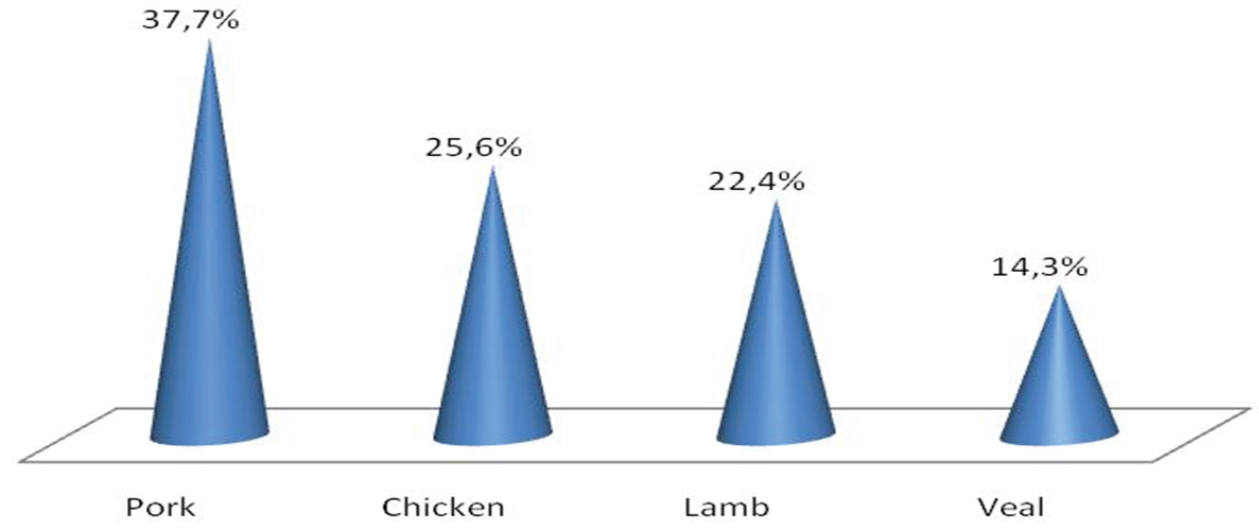

Figure 1: Prevalence of meat food intolerance.

The results show that pork meat intolerance occupies a leading role in the structure of meat intolerance-in 37 people (37.7\%) of all people with meat intolerance. Second in frequency is intolerance to chicken meat- 25 persons $(25.6 \%)$, third place is lamb meat-22 persons $(22.4 \%)$, last in frequency was intolerance to veal meat-14 people (14.3\%).

Correlations between different types of meat. The most often combined intolerance is to:

- Pork and veal-2 persons-2.04\%

- Pork and lamb-1 person-1.02\%

- Lamb and veal-1 person-1.02\%

It is noteworthy that no combined intolerance with chicken meat is found. The intolerance to chicken in the subjects was only found independently.

Correlations between meat (total for all types) and other foods. For a comprehensive analysis of meat intolerance, we sought correlation dependencies with intolerance(s) to other products. Figure 2 presents the most common combined intolerances between meat (all types of meat) and other food groups.

The statistical correlations between intolerance to meat (all types of meat) and intolerance to other products have also been studied (Table 2).

Data shows that persons with meat intolerance (98 persons in total) are most often intolerant to eggs- 83 persons (84.6\%) with strong, statistically significant dependence-p $<0.0001$. This is followed by intolerance to cereals-in 51 people $(52.0 \%)$ and milk-46 people (50.0\%), also with a high degree of confidence $\mathrm{p}<0.0001$, intolerance to nuts-33 persons (33.7\%), gluten-23 persons (23.4\%) and vegetables- 16 $(16.3 \%)(\mathrm{p}>0.001)$

Correlations between different types of meat with other foods (Table 3).

\section{Pork meat}

The most common with statistical significance is the combined intolerance of pork with eggs $\left(\chi^{2}=36.17, \mathrm{p}<0.0001\right)$, corn $\left(\chi^{2}=32.06\right.$, $\mathrm{p}<0.0001)$, fish $\left(\chi^{2}=18.21\right)$, rice $\left(\chi^{2}=12.58, \mathrm{p}<0.0004\right)$. With the other products shown in table 3 there is also dependency, but not statistically significant.

\section{Chicken meat}

The most pronounced, statistically significant dependence was found with: eggs $\left(\chi^{2}=55,23, p<0,0001\right)$, corn $\left(\chi^{2}=50,42, p<0,0001\right)$, yeast $(\chi=28.9, p<0.0001)$, rice $\left(\chi^{2}=25.88, p<0.0001\right)$, rye $\left(\chi^{2}=20.1\right.$, $\mathrm{p}<0.0001)$, milk $\left(\chi^{2}=17.5, \mathrm{p}<0.0001\right)$, and to a lesser extent cashew $\left(\chi^{2}=10.8, p<0.001\right)$. The table also shows intolerance to other types of products, but it has a lower degree of correlation, with no statistically significant reliability.

Lamb meat-the predominant combined intolerance is to eggs $\left(\chi^{2}=36.17, \mathrm{p}<0.0001\right)$, corn $\left(\chi^{2}=54.72, \mathrm{p}<0.0001\right)$, milk $\left(\chi^{2}=48.3\right.$, $\mathrm{p}<0,0001)$, yeast $\left(\chi^{2}=32.44, \mathrm{p}<0.0001\right)$, rice $\left(\chi^{2}=29.24, \mathrm{p}<0.0001\right)$, rye $\left(\chi^{2}=23.13, p<0.0001\right)$. Of lower significance is the combined intolerance between lamb and cashew nuts $\left(\chi^{2}=13.21, p<0.0003\right)$ and almonds $\left(\chi^{2}=10.15, \mathrm{p}<0.0014\right)$. Other products are not statistically significant.

Veal meat-it has the most statistically significant combined intolerances with: eggs $\left(\chi^{2}=79.5, \mathrm{p}<0.0001\right)$, corn $\left(\chi^{2}=74.1, \mathrm{p}<0.0001\right)$, yeast $\left(\chi^{2}=48.97, \mathrm{p}<0.0001\right)$, rice $\left(\chi^{2}=45.21, \mathrm{p}<0.0001\right)$, rye $\left(\chi^{2}=37.89\right.$, $\mathrm{p}<0.0001)$, milk $\left(\chi^{2}=34.48, \mathrm{p}<0.0001\right)$, almonds $\left(\chi^{2}=21.22, \mathrm{p}<0.0001\right)$, soybean $\left(\chi^{2}=21.2, p<0.0001\right)$, legumes $\left(\chi^{2}=17.42, p<0.0001\right)$.

\section{Discussion}

The most consumed meats in Bulgaria are pork (about $10.2 \mathrm{~kg}$ per person/year) and chicken (12.0 kg/person/year) which can be associated with their highest frequency of meat intolerances in the subjects. About $7.7 \mathrm{~kg}$ of minced meat/person was consumed in 2020. The consumption of lamb $(1.2 \mathrm{~kg} /$ person/year $)$ and veal $(1.1$ $\mathrm{kg} /$ person/year) is lower, related to the eating habits of the Bulgarian population, as well as to the relatively higher prices compared to other types of meat $[13,14]$.

The results of our study show that the persons who have IgG mediated meat intolerance are statistically significantly more likely to have intolerance to other foods-correlations, especially (mostly) with eggs, cereals and milk. Chicken, lamb and veal meat show similar trends, while pork meat is different from them: pork meat shows the least tendency to cross-intolerance with other products (eggs, corn and fish), while chicken and lamb show a wider range of reliable crossIgG mediated intolerances-with eggs, milk, corn, wheat, rye, legumes and 


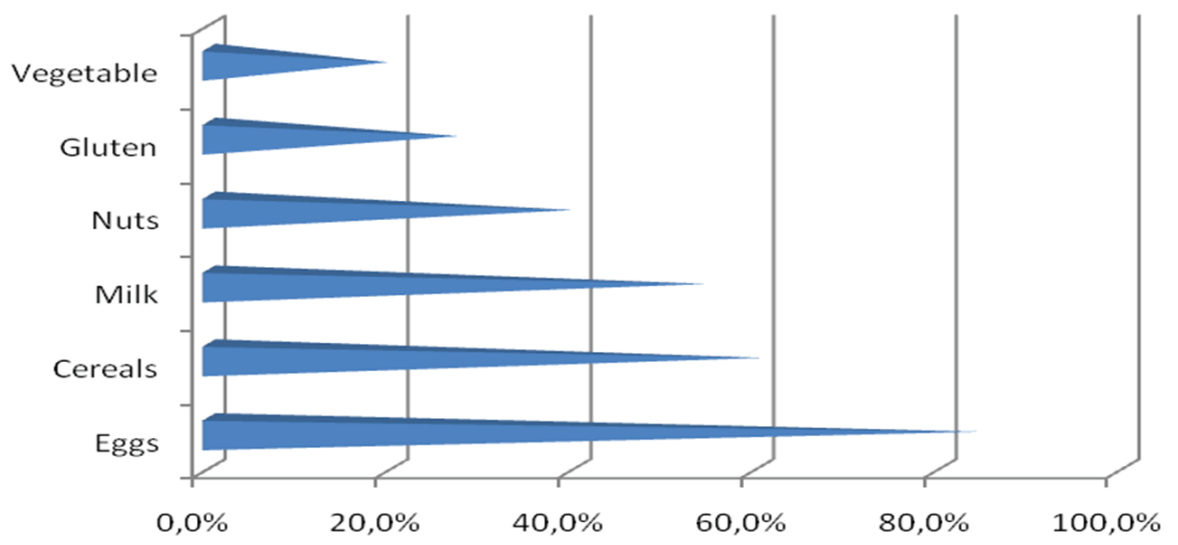

Figure 2: Accompanying food intolerances in people intolerant to meat (\%).

Table 2: Statistical correlations between intolerance to meat (total for all types of meat) and intolerance to other foods.

\begin{tabular}{|l|c|c|c|c|c|c|}
\hline \multicolumn{1}{|c|}{ Intolerance } & Eggs & Cereals & Milk & Nuts & Gegetable \\
\hline \multicolumn{7}{|c|}{ Meat (total for all types of meat) } \\
\hline$\chi^{2}$ & 94.41 & 98.287 & 32.25 & \multicolumn{2}{|c|}{1.635} & 0.12 \\
\hline$p$ & $<0.0001$ & $<0.0001$ & $<0.0001$ & 0.2 & 0.988 \\
\hline$C l$ & $46.89-66.09$ & $27.38-40.02$ & $20.33-44.26$ & & 0.0079 \\
\hline
\end{tabular}

etc.), while veal meat has the widest range of correlations (Table 3). We also found cross-IgG mediated intolerance between different types of red meat, associated primarily with the presence of the same or similar immunological characteristics in different meats.

Chicken meat intolerance is not combined with intolerance to red meats, maybe this is related both to its immunological characteristics and to the way in which the chicken is fed, using food additives, growth hormones, etc., which pass into the meat and are additional allergenic substances [15].

Foods intolerance, i.e. meat intolerance is associated with the release and effect of substances-mediators, which most often can be serum albumins and gamma globulins. In this case intolerance between meat and other products is most commonly associated with crossreactivity to the serum albumins of meat and the respective foods. In case of intolerance to chicken meat, a cross reaction with a-livetin from egg yolk is often detected and cross intolerance is observed. High similarity ( 65\%) of allergens in chicken meat (LMW proteins of 5-25 $\mathrm{kDa}$; light chains of myosin, including $23 \mathrm{kDa}$ MLC-1 (Gal d 7) and $15 \mathrm{kDa}$ MLC-3) with allergens in fish and shrimp was found, whereby persons intolerant to chicken meat show a cross-intolerance to fish and shellfish [16].

In many cases, food/meat intolerance is in a relationship with histamine sensitivity, often associated with a deficiency of the enzyme Diamine oxidase, and occurs in foods rich in histamine or tyramine, foods that enhance histamine synthesis, histamine-releasing foods. Meat is among the foods that contain biologically active substances such as histamine and serotonin, as the meats with the highest histamine content are pork and chicken. Other such foods are fish, cheese, eggs, sausages, berries, pineapple, chocolate, tomatoes, etc.
These intolerances are dose-dependent-the more consumed, the more violent the reaction and some of the cross-reactions in intolerance to pork and chicken with other products containing or triggering elevated histamine levels are also based on this mechanism. Other substances in meat that tend to cause IgG mediated food intolerance can be antibiotics, various food supplements such as coloring agents (erythrosine), preservatives (nitrites), emulsifiers, enzymes and more.

IgG-mediated nutritional intolerance is thought to be caused by increased intestinal permeability, also called "Leaky Gut Syndrome" (LGS), which allows the nutrients to gain access to the bloodstream to form circulating immune complexes and trigger production of specific IgG antibodies. Barrier protection is achieved by non-immune and immune mechanisms. Physiologically (without the involvement of the immune system), this is done by blocking the ingress of the absorbed allergens from the glycocalyx, from the movement of the intestinal epithelium and peristalsis or by destroying the fallen food allergens from hydrochloric acid and pepsin, pancreatic and intestinal enzymes of the intestinal epithelial cells. Immunological protection is accomplished by blocking the passage of received food allergens into the intestinal lumen from antigen-specific secretory $\operatorname{IgA}$, and by removing the food allergens from specific IgA, IgG and phagocytic components of intestine. The production of food-specific IgG antibodies, coupled with the decreased production of anti-inflammatory cytokines such as IL-2,4,5,10,13 and TGF $\beta 1$, is relevant to irritable bowel syndrome [7].

The results of this study show that, in general, food intolerance is more common in women than in men, and so is the meat intolerance. Similar results have been found in other studies [4,17] women are more likely to have food hypersensitivity reactions than men. The results also revealed that females are more likely to have "leaky guts" 
Table 3: Statistical correlations between intolerance to different types of meat with intolerance to other foods.

\begin{tabular}{|c|c|c|c|c|c|c|c|c|c|c|c|}
\hline & Rice & Corn & Rye & Eggs & Milk & Yeast & Wheat. almonds & Cashew & Fish & Legumes & Soy \\
\hline \multicolumn{12}{|c|}{ Pork meat } \\
\hline$x^{2}$ & 12.58 & 32.06 & 8.53 & 36.17 & 6.81 & 14.83 & 1.52 & 2.89 & 18.21 & 0.596 & 1.52 \\
\hline$P$ & 0.0004 & $<0.0001$ & 0.003 & $<0.0001$ & 0.009 & 0.0001 & 0.216 & 0.089 & $<0.0001$ & 0.44 & 0.216 \\
\hline \multicolumn{12}{|c|}{ Chicken meat } \\
\hline$x^{2}$ & 25.883 & 50.42 & 20.1 & 55.23 & 17.5 & 28.9 & 8.1 & 10.8 & 7.55 & 5.72 & 8.1 \\
\hline $\mathrm{P}$ & $<0.0001$ & $<0.0001$ & $<0.0001$ & $<0.0001$ & $<0.0001$ & $<0.0001$ & 0.0044 & 0.001 & 0.006 & 0.016 & 0.004 \\
\hline \multicolumn{12}{|c|}{ Lamb meat } \\
\hline$x^{2}$ & 29.24 & 54.72 & 23. 13 & 59.66 & 20.36 & 32.44 & 10.15 & 13.21 & 5.82 & 7.47 & 10.15 \\
\hline$P$ & $<0.0001$ & $<0.0001$ & $<0.0001$ & $<0.0001$ & $<0.0001$ & $<0.0001$ & 0.0014 & 0.0003 & 0.016 & 0.006 & 0.001 \\
\hline \multicolumn{12}{|c|}{ Veal meat } \\
\hline$x^{2}$ & 45.21 & 74.11 & 37.89 & 79.5 & 34.48 & 48.97 & 21.22 & 25.35 & 0.845 & 17.42 & 21.2 \\
\hline$P$ & $<0.0001$ & $<0.0001$ & $<0.0001$ & $<0.0001$ & $<0.0001$ & $<0.0001$ & $<0.0001$ & $<0.0001$ & 0.358 & $<0.0001$ & $<0.0001$ \\
\hline
\end{tabular}

(Leaky Gut Syndrome (LGS)) predisposing them to the development of food intolerance more frequently than males. In addition, the data are showing that there is no gender difference in the development of IgG-mediated nutritional intolerance [17].

Even in healthy adults, it has been found that about $2 \%$ of the food allergens taken are absorbed in an immunologically active form and can reach all body organs. It happens most quickly in the small intestine, colon and rectum, and more slowly in the esophagus and stomach. Normally in healthy people this does not cause allergic reactions, thanks to the so-called "oral (immunological) tolerance" [18]. High gastric acidity suppresses the absorption of food allergens, and alcohol consumption and hypoacidity have the opposite effect. It has been found that in IgG related food intolerant individuals is easier to overcome the protective mechanisms of the intestinal barrier and create conditions for the rapid passage of larger allergens, increasing hypersensitivity reactions to the food consumed [19].

IgG mediated food intolerance and anti-food IgG antibodies are closely related to overweight and obesity. On one side-the obese individuals showed a highly significant increase in anti-food IgG antibody concentrations, elevated C-reactive protein, compared to normal weight individuals, and on the other side-anti-food $\operatorname{IgG}$ is path genetically involved in the development of obesity and atherosclerosis [11].

We consider, as well as other authors [4], is recommended to study the spread of food-specific IgG antibodies in an asymptomatic population, and in overweight or obese individuals (as the present study was conducted) in order to establish genetic predispositions for weight gain or obesity, specific reference ranges of different nutritional intolerances, individual characteristics and the risk of other health effects. The establishment of IgG mediated nutritional/meat intolerance in individuals allows to adjust the diet, make an eliminatediet, without "intolerable" foods and replace with others, etc. $[8,9,20]$.

\section{Conclusion}

The analysis of the data shows that there are significantly more studies and data on food allergies, while food intolerances have not been sufficiently studied; they are to some extent "underestimated" as significance. Food intolerances create many "hidden" and "overt" manifestations, symptoms, health and nutritional problems.

It is necessary to establish more data on distribution of food intolerances, i.e. IgG mediated meat intolerance and the identification of specific distribution characteristics for the different populations. It should be noted that differences in cultural and eating habits in different parts of the world contribute to variations in the prevalence of food-specific IgG antibodies.

Food intolerances to meat are not among the most common, but they create serious difficulties for those affected (sensitive persons), especially if the meat is main food in the eating habits of the population. Also, food intolerance to meat shows significant correlations with other food intolerances and this further complicates therapeutic behavior and/or dietary prescriptions.

The results of our study show that the persons who have IgG mediated meat intolerance are statistically significantly more likely to have intolerance to other foods-especially (mostly) with eggs, cereals and milk, a lesser extent to vegetables and with no statistical significance for nuts and gluten.

The combination of several intolerances creates significant difficulties in the diet and lifestyle of the affected persons. The importance of $\operatorname{IgG}$ mediated nutritional intolerance is great in the presence of overweight and obesity, and in the presence of chronic diseases, and creates difficulties in normalizing the weight of these individuals and improving their health. The establishment of the IgG mediated nutritional intolerance also contributes to optimizing the diet at the individual level, prescribing healthy individual diets and maintaining the health of the individuals.

In prophylactic terms, determination of food intolerances to meat should be carried out in healthy subjects, who do not report any specific symptoms and/or complaints, as well as in overweight or obese individuals.

The results of the present study can be used in the prevention and treatment of nutritional diseases, the creation of a suitable individual diet, or as an indication for restrictions on the use of particular food groups or products. Very important are the data on possible 
intolerance to meat in patients with impaired metabolism, overweight and other eating disorders, in which proper nutrition is a key factor in the treatment and prevention of the condition.

\section{Availability of Data and Materials}

The datasets used and/or analyzed during the present study available from the author on reasonable request.

\section{Ethics Approval and Consent to Participate}

All subjects were informed and agreed with the present study. No personal data which could identify the subjects were collected for this study. All health data were used only for statistical purposes.

\section{Human and Animal Rights}

No animals were used for studies that are the basis of this research. All humans participated in the tests voluntarily.

\section{Consent for Publication}

All subjects who accepted to be tested for the study were informed and participated voluntarily.

\section{Conflict of Interests}

The authors declare no conflict of interest, financial or otherwise.

\section{References}

1. Zeng Q, Dong SY, Wu LX, Li H, Sun ZJ, et al. (2013) Variable foodspecific IgG antibody levels in healthy and symptomatic Chinese adults. PLoS One 8: e53612.

2. Priedite V, Nikiforenko J, Kurjanev N, Kroica J (2014) Antigen Specific IgG4 in Patients with Gastrointestinal Complaints. Brit J Med \& Med Res 4: 194-201.

3. Gaur S, Kumar R (2013) Food allergy or food intolerance? Indian J Allergy Asthma Immunol 27: 93.

4. Shakoor Z, AlFaifi A, AlAmro B, AlTawil LN, AlOhaly RY (2016) Prevalence of IgG-mediated food intolerance among patients with allergic symptoms. Ann Saudi Med 36: 386-390.

5. Collard J (2010) Food Allergy and Intolerance. Pract Nurse 39: 17-21.

6. Kumar R, Singh M, Gupta N, Kumar M, Bisht I, et al. (2013) Prevalence of food intolerance in bronchial asthma in India. Indian J Allergy Asthma Immunol 27: 121-128.

7. Sentsova TB, Vorozhko IV, Isakov VA, Morozov SV, Shakhovskaia AK (2014) Immune status estimation algorithm in irritable bowel syndrome patients with food intolerance. Eksp Klin Gastroenterol 7: 13-17.
8. Guo H, Jiang T, Wang J, Chang Y, Guo H, et al. (2012) The Value of Eliminating Foods According to Food-Specific Immunoglobulin G Antibodies in Irritable Bowel Syndrome with Diarrhoea. J Int Med Res 40: 204-210.

9. Mitchell N, Hewitt CE, Jayakody S, Islam M, Adamson J, et al. (2011) Randomised controlled trial of food elimination diet based on IgG antibodies for the prevention of migraine like headaches. Nutr J 10: 85.

10. Arroyave Hernández $\mathrm{CM}$, Echavarría Pinto $\mathrm{M}$, Hernández Montiel HL (2007) Food allergy mediated by IgG antibodies associated with migraine in adults. Rev Alerg Mex 54: 162-168.

11. Wilders-Truschnig $\mathrm{M}$, Mangge $\mathrm{H}$, Lieners $\mathrm{C}$, Gruber $\mathrm{H}$, Mayer $\mathrm{C}$, et al. (2008) IgG antibodies against food antigens are correlated with inflammation and intima media thickness in obese juveniles. Exp Clin Endocrinol Diabetes 116: 241-245.

12. Food Detective IgG Food Intolerance Test-CNS (Cambridge Nutrition Sciences Ltd).

13. National Statistical Institute-Republic of Bulgaria.

14. Institute of Market Economics. Market analysis for meat products and meat consumption in Bulgaria.

15. Turner PJ, Kemp AS (2012) Intolerance to food additives-does it exist? J Paediatr Child Health 48: E10-E14.

16. Hemmer W, Klug C, Swoboda I (2016) Update on the bird-egg syndrome and genuine poultry meat allergy. Allergo J Int 25: 68-75.

17. Harish Babu BN, Mahesh PA, Venkatesh YP (2008) A crosssectional study on the prevalence of food allergy to eggplant (Solanum melongena L.) reveals female predominance. Clinical and experimental allergy: J Brit Soci Allergy \& Clin Immunol 38: 17951802.

18. Chang LR, Li FY, Li WS, Jiang YM (2013) An epidemiological study of food intolerance in 2434 children. Zhongguo Dang Dai Er Ke Za Zhi 7: 550-554.

19. Burks AW, Laubach S, Jones SM (2008) Oral tolerance, food allergy, and immunotherapy: implications for future treatment. J Allergy Clin Immunol 121: 1344-1350.

20. Aydinlar El, Dikmen PY, Tiftikci A, Saruc M, Aksu M, et al. (2013) IgGbased elimination diet in migraine plus irritable bowel syndrome. Headache 53: 514-525. 